\title{
Information der SAGOM
}

Manuelle Medizin 2017 · 55:326

https://doi.org/10.1007/s00337-017-

0314-0

C) Springer Medizin Verlag GmbH 2017

V.i.S.d.P.:

Dr. B. Weber

Bahnhofstrasse 63

$\mathrm{CH}-8887$ Mels

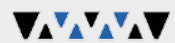 \\ OSTEOPATHISCHEMEDIZIN \\ SAGOM \\ Schweizerische Ärztegesellschaft \\ für Osteopathische Medizin SAGOM
}

\section{Curriculum Kurse der SAGOM 2017 \\ (In Kooperation mit DGOM) \\ Die Kurse finden alle in Freiburg (D) statt}

13.01.-15.01. Cranio 3 Kenntnisvoraussetzung: Cranio 2, Parietale Integration

17.02.-19.02. Grundlagenkurs für Osteopathische Medizin GKOM/T

Eingangsvoraussetzung:

Ärzte: Abgeschlossene Weiterbildung in manueller Medizin.

Physiotherapeuten: Nachweis einer manual-therapeutischen Weiterbildung.

07.07.-09.07. Cranio 2

Kenntnisvoraussetzung: Cranio 1

08.09.-10.09. MET 1

Kenntnisvoraussetzung: GKOM/T

15.09.-17.09. Viszerale Techniken VIS 1

Kenntnisvoraussetzung: Anatomie

22.09.-24.09. Counterstrain CoSt

Kenntnisvoraussetzung: GKOM/T

\section{Masterkurse der SAGOM 2017}

(In Kooperation mit DGOM)

Die Kurse finden alle in Freiburg (D) statt

07.04.-09.04. Mechanical link 1

(nach neuem Curriculum 2016)

(Nach Paul Chauffour D. O.) Kursleiter: Eric Prat,

D. O. F/D

Kenntnisvoraussetzung: Diplom D. O.M./D O.T

oder abgeschlossene Osteopathische Fortbildung bei einer anerkannten Schule mit Diplom Abschluss.

02.02-05.02. Mechanical link 4

(nach neuem Curriculum 2016)

(Nach Paul Chauffour D. O.)

Kursleiter: Claudia Hafen, MSc D. O., GDK Kenntnisvoraussetzung: LMO 1

30.06.-02.07. Mechanical link 5

(Nach Paul Chauffour D.O.)

Kursleiter: Claudia Hafen, MSc D. O., GDK

Kenntnisvoraussetzung: LMO 1

01.12.-03.12. Mechanical link 6

(Nach Paul Chauffour D. O.)

Kursleiter: Eric Prat, D. O. F/D

Kenntnisvoraussetzung: LMO 1

\section{Anmeldungen:}

DGOM Sekretariat

c/o Akademie für Gesundheitsfachberufe

am Diakonie Krankenhaus Belchenstraße 1 -5, 68163 Mannheim

Telefonnummer des Sekretariats: 0621/436266 92

Faxnummer: 0621/436266 91

Email: kontakt@dgom.info

Ansprechpartnerin in allen Fragen zu DGOM Kursen ist Frau Citius.

\section{SAGOM Vereinssekretariat:}

SAGOM Sekretariat

c/o Praxis Dr. med. Bruno Weber Bahnhofstrasse 63

$\mathrm{CH} 8887$ Mels

Tel +41817200540

Fax +41 817200545

E Mail: info@sagom.ch www.sagom.ch

Bitte beachten Sie auch das Curriculum der DGOM. 